\title{
Malrotación intestinal en adultos: causa infrecuente de obstrucción intestinal
}

\author{
Intestinal malrotation in adults: An uncommon cause of intestinal \\ obstruction

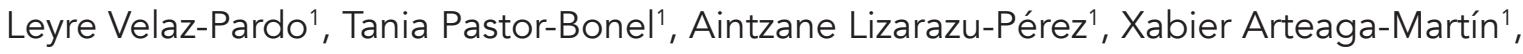 \\ Patrizio Petrone ${ }^{2}$ \\ 1 Servicio de Cirugía General y del Aparato Digestivo, Hospital Universitario Donostia, Donostia-San Sebastián, Guipúzcoa, España. \\ 2 Director of Surgical Research, Department of Surgery, NYU Winthrop Hospital: Mineola, New York, NY, USA; Associate Professor \\ of Clinical Surgery, NYU Long Island School of Medicine: Mineola, Stony Brook, New York, NY, USA \\ Póster presentado en la XXI Reunión Nacional de Cirugía en Málaga, España, octubre de 2017.
}

\section{Resumen}

La malrotación intestinal es una malformación congénita que afecta hasta al I \% de la población. Aproximadamente, el $90 \%$ de los casos se presenta en la edad pediátrica y, rara vez, en la población adulta, lo que convierte a esta alteración en un reto para los profesionales sanitarios.

Se presenta el caso de una paciente que se inició con un cuadro de obstrucción intestinal y abdomen agudo; se le diagnosticó malrotación intestinal, vólvulo y obstrucción por bridas, durante la laparotomía exploradora urgente. El conocimiento de condición patológica es imprescindible para poder brindarle un correcto tratamiento quirúrgico.

Palabras clave: abdomen agudo; obstrucción intestinal; vólvulo intestinal; anomalías congénitas.

\begin{abstract}
The intestinal malrotation is a congenital malformation that affects up to $\mathrm{I} \%$ of the population. Approximately $90 \%$ of cases are diagnosed in the pediatric age and rarely in the adult population, which makes this pathology a challenge for health professionals.

This is a case of a patient who presented with intestinal obstruction and acute abdomen that was diagnosed during the emergency exploratory laparotomy with intestinal malrotation, volvulus and obstruction. The knowledge of this pathology is essential to perform its correct surgical treatment.
\end{abstract}

Keywords: Intestinal malrotation; intestinal obstruction; Ladd's procedure.

Fecha de recibido: 2/11/2018 - Fecha de aceptación: 28/12/2018

Correspondencia: Leyre Velaz-Pardo, MD, Hospital Universitario Donostia, Paseo Dr. Beguistain 109, CP 20014, San Sebastián, Guipúzcoa, España. Teléfono: (+34) (600) 79-8878

Correo electrónico: Ivelaz93@gmail.com

Citar como: Velaz-Pardo L, Pastor-Bonel T, Lizarazu-Pérez A, Arteaga-Martín X, Petrone P. Malrotación intestinal en adultos: causa infrecuente de obstrucción intestinal. Rev Colomb Cir. 2019;34:287-91. https://doi.org/10.30944/20117582.444

Este es un artículo de acceso abierto bajo una Licencia Creative Commons - BY-NC-ND https://creativecommons.org/licenses/by-nc-nd/4.0/deed.es 


\section{Introducción}

La malrotación intestinal es una malformación congénita que afecta hasta al I \% de la población. Aproximadamente, el $90 \%$ de los casos se presenta en la edad pediátrica y, rara vez, en la población adulta ${ }^{\text {. }}$.

Su presentación varía desde un cuadro clínico de abdomen agudo debido a la isquemia aguda por un vólvulo del intestino delgado, hasta síntomas digestivos crónicos, como dispepsia, náuseas, vómitos, estreñimiento y dolor de tipo cólico $^{2,3}$.

\section{Caso clínico}

Se trata de una paciente de 32 años de edad que acudió al servicio de urgencias por presentar dolor abdominal generalizado, estreñimiento y vómitos de seis días de evolución; además, pérdida de peso. Había sido estudiada previamente en el Servicio del Aparato Digestivo por presentar síntomas similares desde la infancia, aunque de resolución espontánea y sin haberse establecido la causa. Durante la exploración clínica, se observó distensión abdominal, molestias abdominales generalizadas e irritación peritoneal localizada en la fosa ilíaca derecha.

En el Servicio de Urgencias se solicitó una ecografía abdominal, en la cual se observó una lesión quística de $2 \mathrm{~cm}$ de diámetro correspondiente a un quiste del uraco e ileítis. Tras dichos hallazgos, la paciente ingresó a cargo del Servicio del Aparato Digestivo donde, dada la ausencia de mejoría del cuadro clínico, se ordenó una tomografía computarizada (TC) de abdomen y pelvis. En la misma, se identificaron cambios inflamatorios de la grasa mesentérica, y la raíz vascular del mesenterio se encontró rodeada por un manguito de tejido, correspondiente a una brida, además del tronco arterial común del que nacen la arteria gástrica izquierda, la hepática y la mesentérica superior, y la vena mesentérica superior que drena en la vena porta izquierda, conformando el signo del remolino (whirlpool sign) ${ }^{4}$, y un probable quiste de uraco de $18 \mathrm{~mm}$ de diámetro (figuras I y 2).

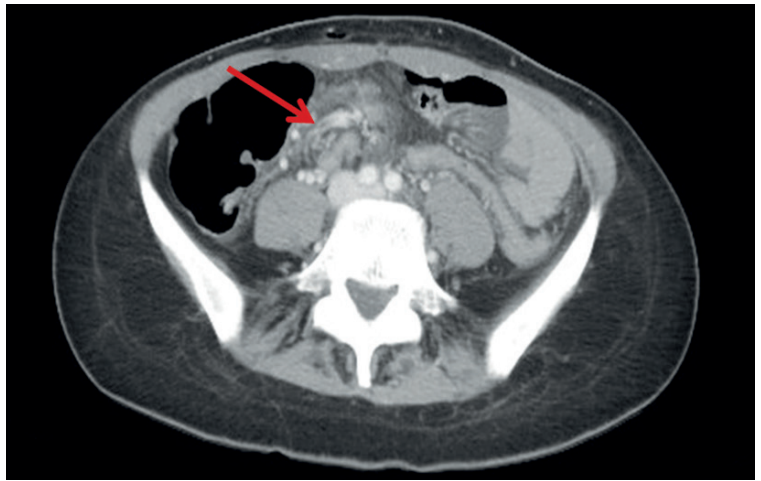

Figura 1. Se observan los vasos arteriales arremolinados alrededor de la base del pedículo mesentérico. Este es el signo del remolino, visible en la tomografía computarizada cuando la malrotación intestinal se asocia con vólvulo del intestino delgado.

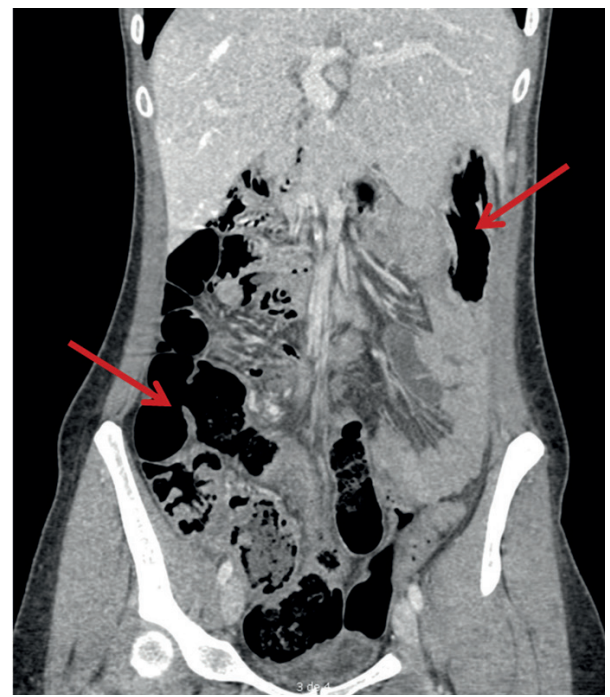

Figura 2. Las flechas señalan el colon en el hemiabdomen derecho y el estómago en el flanco izquierdo; el marco cólico y el ángulo de Treitz no se observan.

Debido a la presentación clínica de la paciente y a la exploración abdominal patológica, además del aumento de la irritación peritoneal respecto al día anterior, se decidió la intervención quirúrgica de forma urgente. Se practicó una laparotomía media supraumbilical e infraumbilical, y se encontró una malrotación intestinal completa con una brida de grueso calibre insertada en el mesenterio del colon derecho y en el ombligo. Sobre ese ligamento, se produjo la rotación del colon y del intestino delgado; no había signos de 
sufrimiento intestinal. Se constató la ausencia del ligamento redondo y la presencia de adherencias entre el duodeno y el ciego (figura 3).

Se seccionó la brida y se liberaron las adherencias, manteniendo la posición original del intestino delgado y del colon, es decir, sin intentar colocarlos en la ubicación anatómica habitual, y se llevó a cabo la apendicectomía (figura 4). Tras el procedimiento, la paciente evolucionó favorablemente, y fue dada de alta a los tres días de la intervención quirúrgica. Al cabo de un año, la paciente refirió ausencia de sintomatología digestiva, con buena tolerancia de la vía oral y aumento ponderal.

\section{Discusión}

La malrotación intestinal se debe a una falla de la rotación intestinal y su fijación retroperitoneal durante el desarrollo embrionario. La rotación intestinal sucede en tres etapas, descritas por primera vez por Frazer y Robbins en 1915 5,6. Según el

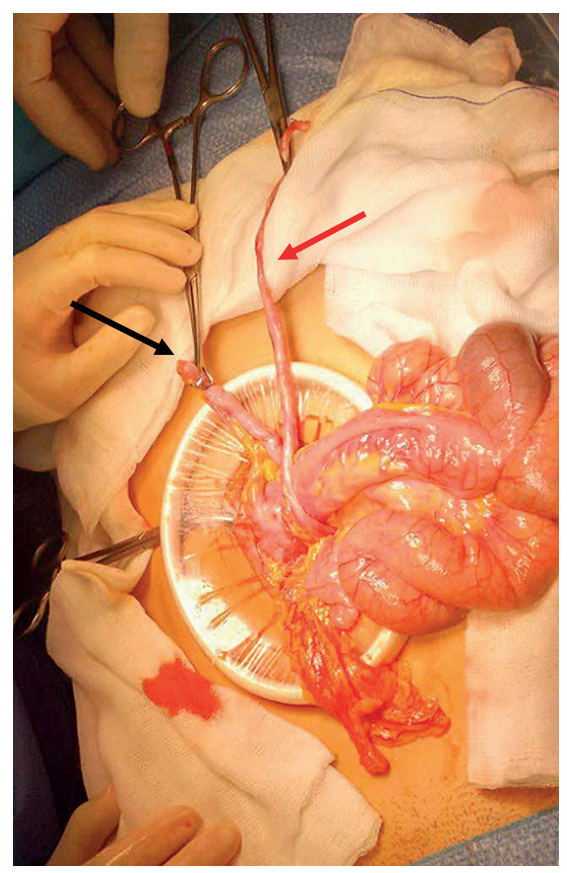

Figura 3. Se señalan el apéndice cecal en el hipocondrio derecho (flecha negra) y una brida insertada en el mesocolon derecho (flecha roja). momento el que se interrumpe dicha rotación, se producen diferentes tipos de posición intestinal anormal en el abdomen ${ }^{7,8}$.

Las anomalías de la rotación del intestino medio se clasifican como sigue:

- IA. No se produce la primera rotación intestinal de $90^{\circ}$ en sentido antihorario (levógira). Se puede complicar con vólvulo del intestino medio.

- IIA. Ocurre durante la segunda etapa de rotación intestinal, que es levógira y de $180^{\circ}$. El duodeno no rota, mientras que el colon lo hace de forma normal. Se puede complicar con obstrucción duodenal por bandas de Ladd.

- IIB. Afecta también la segunda etapa de rotación, normalmente levógira y de $180^{\circ}$. El duodeno y el colon rotan en sentido inverso, o sea, dextrógiro. Se puede complicar con obstrucción del colon transverso por el mesenterio duodenal.

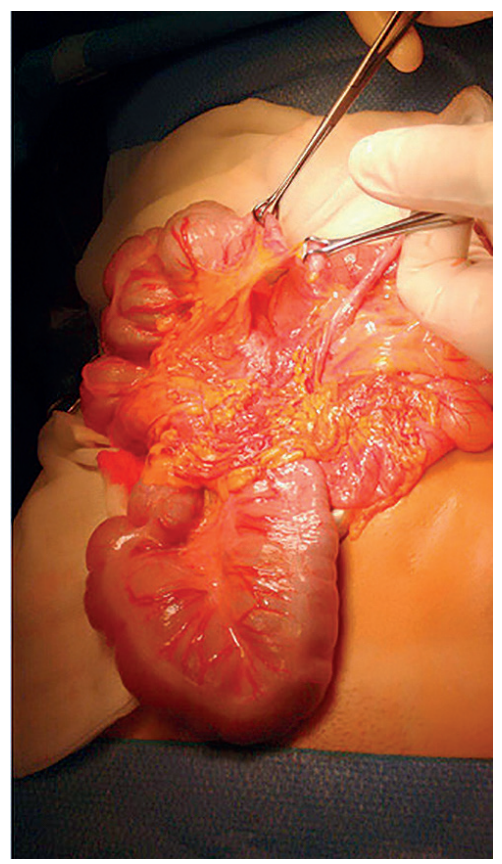

Figura 4. Después de seccionar la brida, se observan el ciego y el colon sigmoide en el hemiabdomen derecho; el ciego, con su apéndice, se encuentran en el hipocondrio derecho. 
- IIC. Durante la segunda etapa de rotación, mientras el colon rota $180^{\circ}$ en sentido levógiro, lo cual es normal, el duodeno rota en sentido contrario (dextrógiro). Esto hace que se cree una bolsa mesentérica derecha que puede actuar como un saco herniario.

- IIIA. Durante la tercera etapa embrionaria, no se produce la rotación normal, levógira y de $180^{\circ}$ del colon, aunque el duodeno sí rota normalmente. Se puede complicar con vólvulo del intestino medio.

- IIIB. Durante la tercera etapa embrionaria, el mesenterio del ángulo hepático del colon no se fija completamente y se produce una rotación levógira, pero de $270^{\circ}$. Puede complicarse con obstrucción por bandas de Ladd.

- IIIC. Durante la tercera etapa embrionaria, se produce una alteración similar a la anterior, pero la falta de fijación del mesenterio afecta al ciego y al sigmoide. Puede complicarse con vólvulo del ciego, invaginación, o ambas. La asociación de invaginación y malrotación intestinal recibe el nombre de síndrome de Waugh.

- IIID. Sucede también durante la tercera etapa embrionaria, porque la fijación del mesenterio es incompleta y se producen hernias internas. Puede complicarse con una hernia paraduodenal.

Dados los hallazgos intraoperatorios de rotación inversa del duodeno y del colon, este caso clínico se puede clasificar como una malrotación intestinal de tipo IIB.

La paciente había presentado síntomas digestivos de forma crónica. Este episodio inició como un cuadro clínico de obstrucción intestinal, cuya evolución indicaba la presencia de isquemia aguda, por lo que se decidió la intervención quirúrgica urgente. Si bien los hallazgos descritos en la TC previa ya eran sugestivos de malrotación intestinal y vólvulo, el diagnóstico definitivo se hizo durante la cirugía mediante la observación directa del intestino.

En la edad adulta, la malrotación intestinal puede ser asintomática y diagnosticarse de forma incidental, se puede presentar con síntomas crónicos o puede manifestarse por primera vez con un cuadro clínico de abdomen agudo. Según las diferentes series de casos reportadas, en los pacientes con manifestaciones clínicas, los síntomas son crónicos en la mitad de los casos, aproximadamente, y de inicio agudo, en la otra mitad.

El examen de elección para diagnosticar la malrotación intestinal continúa siendo el tránsito intestinal ${ }^{7,9}$, con una sensibilidad del 93 al Ioo \% y una especificidad cercana al $85 \%$, aunque la sensibilidad para el diagnóstico de vólvulo es solo del $53 \%$. El diagnóstico se basa en comprobar la posición anómala de la unión duodeno-yeyuno, desplazada inferior y medialmente.

La ecografía es menos sensible (67 a Ioo \%) y menos específica (75 a $83 \%$ ) para el diagnóstico de malrotación, aunque es un examen de bajo costo económico que permite su diagnóstico precoz. Además, es el método diagnóstico estándar cuando se sospecha un vólvulo del intestino medio, ya que tiene una sensibilidad del $92 \%$ y una especificidad del roo \%; el signo del remolino es el más característico ${ }^{7,9}$. La mayoría de los adultos se diagnostican mediante TC abdominal, con contraste oral o sin él, la cual tiene una exactitud diagnóstica del $80 \%{ }^{9}$; además, permite diagnosticar anomalías anatómicas en otros órganos, como la hipoplasia del proceso unciforme del páncreas, inducida por la interferencia de la rotación del primordio pancreático, el cual normalmente rota junto con el asa duodeno-yeyuno ${ }^{7}$. Las mismas ventajas las puede ofrecer la resonancia magnética (RM). En la edad pediátrica, es preferible evitar la práctica de la TC por la alta dosis de radiación y, la de la RM, por su baja resolución ?

Ante la sospecha de vólvulo o de isquemia, el tratamiento indicado es la intervención quirúrgica urgente, ya sea mediante laparotomía o laparoscopia, para llevar a cabo la cirugía de Ladd ${ }^{\text {Io. }}$. Este procedimiento consiste en reducir el vólvulo de intestino delgado, si está presente, seccionar las bandas ('adhesiólisis') de Ladd y proceder a la apendicectomía cuando el apéndice cecal esté en una posición anómala " 
último, porque su diagnóstico sería difícil en caso de desarrollarse después una apendicitis ${ }^{12}$. La cecopexia no se recomienda, ya que no se ha demostrado que disminuya la morbimortalidad posoperatoria ${ }^{3}$.

Si no se procede a una intervención quirúrgica oportuna, el paciente podría sufrir complicaciones como la perforación de víscera hueca, la isquemia intestinal o la peritonitis, lo cual aumentaría la morbimortalidad de forma considerable. En casos ya complicados, la recomendación es resolver la enfermedad abdominal aguda en un primer tiempo quirúrgico y practicar la cirugía de Ladd en un segundo tiempo 9 .

Sin embargo, el tratamiento de los pacientes asintomáticos sigue generando controversia. Algunos autores defienden la observación de estos pacientes, ya que parece que tienen menor riesgo de desarrollar un vólvulo, mientras que otros defienden el manejo quirúrgico, ya que es imposible predecir si van a desarrollar o no un vólvulo intestinal junto con sus posibles complicaciones ${ }^{9}$.

\section{Conclusión}

La obstrucción del intestino delgado o la isquemia mesentérica causadas por malrotación intestinal, son infrecuentes. Sin embargo, es de suma importancia conocer y sospechar la malrotación intestinal como su causa, para poder diagnosticarla y tratarla oportunamente, evitándose así la morbimortalidad que puede acarrear.

Conflictos de interés: Ninguno declarado

Fuente de financiación: Autofinanciado

\section{Referencias}

I. Durkin ET, Lund DP, Shaaban AF, Schurr MF, Weber $\mathrm{SM}$. Age-related differences in diagnosis and morbidity of intestinal malrotation. J Am Coll Surg. 2008;206:65863. doi: I0.IOI6/j.jamcollsurg.2007.II.020.

2. Kapfer SA, Rappold JF. Intestinal malrotation -not just the pediatric surgeon's problem. J Am Coll Surg. 2004;199:628-35.

3. Vaos G, Misiakos EP. Congenital anomalies of the gastrointestinal tract diagnosed in adulthood -diagnosis and management. J Gastrointest Surg. 20I0;14:916-25.

4. Yang B, Chen W, Zhang XF, Luo ZR. Adult midgut malrotation: Multi-detector computed tomography findings of I4 cases. Jpn J Radiol. 2013;31:328-35.

5. Frazer JE, Robbins RH. On the factors concerned in causing rotation of the intestine in man. J Anat Physiol. I915;50;75-IIO.

6. Roa I, Meruane M. Desarrollo del aparato digestivo. Int J Morphol. 2012;30:I285-94.

7. Ballesteros E, Torremadé A, Durán C, Martín C, Caro A. Actualización: malrotación-vólvulo intestinal: hallazgos radiológicos. Radiología. 20I5;57:9-2I.

8. Guerrero J, Guerrero-Fdez J. Malrotacion intestinal. Web PEDiátrica. Fecha de consulta: 2 de noviembre 2 de 2018. Disponible en: http://www.webpediatrica.com/ casosped/pdf/II7_malrotacion_intestinal.pdf

9. Emanuwa O, Ayantunde A, Davies T. Midgut malrotation first presenting as acute bowel obstruction in adulthood: A case report and literature review. World J Emerg Surg. 20II;6:22. doi: I0.II86/I749-7922-6-22.

Io. Ladd WE. Surgical diseases of the alimentary tract in infants. N Engl J Med. 1936;215:705-8.

II. Kalfa N, Zamfir C, López M, Forgues D, Raux O, Guibal MP, et al. Conditions required for laparoscopic repair of subacute volvulus of the midgut in neonates with intestinal malrotation: 5 cases. Surg Endosc. 2004;18:1815-7.

12. Arévalo C, Owen K, Tabares C, Bernal F, Borráez B. Apendicitis aguda izquierda: presentación infrecuente de una enfermedad frecuente. Revisión de la literatura y reporte de un caso. Rev Colomb Cir. 20I5;30:I46-50. 